\title{
Evidence for Multiple Functional Copies of the Male Sex-Determining Locus, Sry, in African Murine Rodents
}

\author{
Barbara L. Lundrigan, Priscilla K. Tucker \\ Museum of Zoology and Department of Biology, University of Michigan, Ann Arbor, MI 48109-1079, USA
}

Received: 4 October 1996 / Accepted: 17 January 1997

\begin{abstract}
Southern hybridization data suggest that the male sex-determining locus, Sry, is often duplicated in rodents. Here we explore DNA sequence evolution of orthologous and paralogous copies of Sry isolated from six species of African murines. PCR amplification followed by direct sequencing revealed from two to four copies of Sry per species. All copies include a long open reading frame, with a stop codon that coincides closely with the stop codon of the house mouse, Mus musculus, a species known to have a single copy of Sry. A phylogenetic analysis suggests that there are at least seven paralogous copies of Sry in this group of rodents. Putative orthologues are identical; sequence divergence among putative paralogues ranges from 1 to $8 \%$ (excluding the CAG repeat), with much lower levels of divergence in the high-mobility group (HMG-box) region than in the C-terminal region. A high proportion of nucleotide substitutions in both regions result in aminoacid replacement. The long open reading frame, conserved HMG-box, and pattern of evolution of the putative paralogues suggest that they are functional.
\end{abstract}

Key words: $\quad$ Sry - HMG-box - Gene duplication Sex determination - Arvicanthis - Lemniscomys Pelomys - Rhabdomys

\section{Introduction}

In mammals, male sex determination is defined by the initiation of testis development. There is now consider-

Correspondence to: $\mathrm{B}$. Lundrigan able evidence that Sry (sex-determining region $Y$ chromosome) is the testis-determining gene (Gubbay et al. 1990; Sinclair et al. 1990). With rare exception (see, e.g., Bianchi et al. 1993), Sry is male-specific in all mammal species examined thus far, a sample that includes representatives from three metatherian and seven eutherian orders (Sinclair et al. 1990; Foster et al. 1992; Pallsboll et al. 1992; Bianchi and Bianchi 1993). It is expressed in the genital ridge at a time consistent with its having a role in testis determination (Gubbay et al. 1990; Koopman et al. 1991b), and a 14.5-kb mouse genomic DNA fragment containing Sry can induce testis development when injected into (chromosomally female) XX mouse embryos soon after fertilization (Koopman et al. 1991a).

Sry consists of a single exon with a conserved aminoacid DNA-binding domain (high-mobility group or HMG-box) of approximately 79 base pairs (bp) surrounded by $\mathrm{N}$-terminal and $\mathrm{C}$-terminal regions of variable length and composition. The Sry protein binds to specific target DNA sequences, inducing a sharp bend (Nasrin et al. 1991; Ferrari et al. 1992) that is thought to influence the transcription of (still unidentified) genes involved in the differentiation of Sertoli cells. Binding activity resides in the HMG-box and mutations in this region have been associated with sex reversal in humans (reviewed in Hawkins 1993).

The importance of the N-terminal and C-terminal regions to the function of Sry is not known. These nonHMG-box regions differ markedly between species and can be aligned only among closely related taxa. Comparative studies within primates (Whitfield et al. 1993) and rodents (Tucker and Lundrigan 1993) suggest a rapid 
rate of amino-acid sequence evolution in the nonbox regions, indicating that they are either under directional selection or are not functionally constrained.

A single copy of Sry occurs in humans and the house mouse, Mus musculus, but Southern hybridization studies of both New World sigmodontine rodents (Bianchi et al. 1993) and Old World murine rodents (Nagamine 1994) suggest that Sry sometimes occurs in two or more copies. Here we explore DNA sequence evolution of orthologous and paralogous copies of Sry isolated from six species of African murine rodents.

\section{Materials and Methods}

DNA Samples and Sample Preparation. The Arvicanthis division is an African radiation of herbivorous rodents (family Muridae, subfamily Murinae) that includes 14 genera united primarily on the basis of tooth morphology (Misonne 1969). The phylogenetic relationships among the species in this group are not well understood. We sampled two males and two females from each of six species from the Arvicanthis division: Arvicanthis nairobae, Lemniscomys barbarus, L. massaicus, L. rosalia, Pelomys fallax, and Rhabdomys pumilio. Tissues were obtained from field-collected specimens kindly donated by W. N. Verheyen. Genomic DNA was isolated from frozen tissue following the method of Jenkins et al. (1982).

Polymerase Chain Reaction (PCR). Various combinations of forward (5' to $3^{\prime}$ : AGATCTTGATTTTTAGTGTTC, TAGAGAGCATGGAGGGCCAT, TCCTACACAGAGAGAAATACC, GTCTGCTACAGTGGGACATAA, CTCCAAGGCCCAGCAAAGCTT, ACCAGCAGAGCAAAGGACCAT) and reverse (5' to $3^{\prime}$ : TGTACAGTTT (T/C) GTTGAGGC, CACCAGTGATGTCAGCTGTTAGTA, GTATCTAAACCAACTAGGAATCTC, TGTCTGTATGTGA T GGTGTGG, GA GGACAGGTGTGCA GCTCTA, AAGCTTTGCTGGGCCTTGGAG, ATGGTCCTTTGCTTTGCTGGT, CAAGGAGGAGTATTGGCATAGGTA) primers were used to PCR amplify overlapping segments of Sry beginning $76 \mathrm{bp} 5^{\prime}$ to the open reading frame (ORF) and extending slightly beyond the putative stop codon of each sequence, for a total of from 658 to $685 \mathrm{bp}$. The first three forward primers and the first three reverse primers were designed from the published Mus musculus Sry sequence (Gubbay et al. 1992) and are highly conserved across Old World rodent species. The other primers were designed to match Sry of the species being investigated here, and once multiple copies were discovered, to target particular copies of the gene. The amplification protocol included 30 cycles, with denaturation at $95^{\circ} \mathrm{C}$ for $1 \mathrm{~min}$, annealing at from 50 to $65^{\circ} \mathrm{C}$ for $1 \mathrm{~min}$, and elongation at $72^{\circ} \mathrm{C}$ for $1 \mathrm{~min} 15 \mathrm{~s}$. Because the DNAs were degraded, we were unable to explore the number of multiple copies of Sry using Southern hybridization.

To verify that amplified sequences were located on the Y chromosome, amplification experiments included both male and female DNA from each species under investigation. Amplification of the target sequences in males only was taken as evidence that the sequences were Y-specific. PCR products were visualized on a $2 \%$ NuSieve gel. If multiple bands were observed, they were cut from the gel and gene cleaned using the Geneclean II kit (Bio 101). Samples for which only a single band was observed were Centricon cleaned using Centricon 100 tubes (Amicon).

Sequencing and Sequence Alignment. DNA was sequenced using the Sanger dideoxy-sequencing technique (Sanger et al. 1977) and the Cycle Sequencing System (Gibco BRL). Because PCR products sometimes contained more than a single copy of Sry, each PCR product was sequenced several times using several different sequencing primers. Many of our sequences are incomplete, particularly in the $5^{\prime}$ region where the copies are very similar, and it was thus difficult to target and definitively identify particular copies of the gene. Sequences were aligned by eye with the published Mus musculus Sry sequence (Gubbay et al. 1992). The 3' end of the Sry sequences includes an imperfect CAG repeat of variable length, which could not be unambiguously aligned; we excluded that region from the phylogenetic analysis.

Phylogenetic Analysis. Aligned sequences were subjected to a parsimony analysis using PAUP (version 3.1.1, Swofford 1993). Characters were unordered (Fitch optimization) and uniformly weighted. We performed a single analysis, using the branch-and-bound option, with the Mus musculus sequence as an outgroup.

A decay index (Kallersjo et al. 1992) was calculated for each clade. The decay index of a given clade is the number of additional steps required to generate a nonminimal-length tree in which that clade has been dissolved. MacClade (ver. 3.01, Maddison and Maddison 1992) was used to trace character changes and document support for individual branches.

\section{Results}

\section{Identification of Multiple Copies of Sry}

We identified from two to four copies of Sry per species, for a total of 17 sequences. All sequences were malespecific, i.e., no amplification resulted when female DNA was used as a template. An alignment of the variable sites, excluding the CAG repeat region, is presented in Fig. 1.

\section{Parsimony Analysis}

To distinguish orthologous copies of Sry (those resulting from speciation) from paralogous copies (those resulting from gene duplication), the aligned sequences in Fig. 1 were subjected to a parsimony analysis. This analysis resulted in 48 most parsimonious trees with a length of 40 and a consistency index of 0.975 . A strict consensus is shown in Fig. 2.

There is only one homoplasious character on this tree, a nucleotide site with a character state that is shared by the outgroup and the lineage labeled copy 7 . The large number of most parsimonious trees and the low decay index for most clades reflect the large amount of missing data. Missing data also tend to inflate the consistency index, since for missing entries, PAUP assigns the character state that would be most parsimonious given the tree topology.

The consensus tree suggests that there are at least seven paralogous copies of Sry in this group of rodents (copy 1-7 in Fig. 2). Each is diagnosed by three or more unique and unreversed synapomorphies. For copies 1, 2, and 6, some of these diagnostic characters are present in only a single terminal taxon and have been assumed for the other members of the clade, taxa for which the relevant sites are missing. However, even when those characters are not counted as support, copies 1,2, and 6 are 
Mmu aggttgGGATGTGAAGCAGAGGTTCTGACAATCTGATCACA. . . . TTTTCTGATCTGGTAACCC An ?????????????????????????????????????--A- . . . . . TTTTT--A--T-----Ga--

An -......-.

An - -

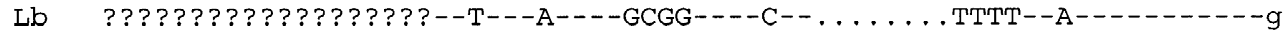

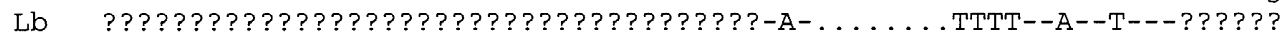

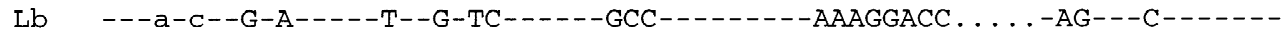

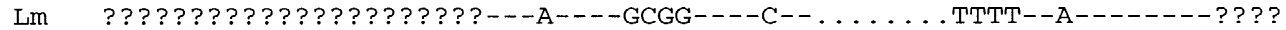

Lm -----A----C-C--T----CC-----GGCC-----A---AAAGGACC . . .-CCG----ACG----

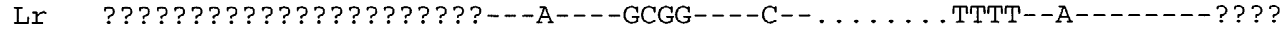

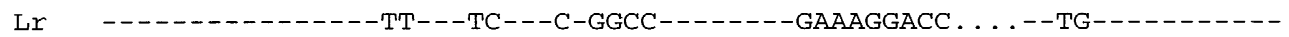

Pf -a--C--A-------T----T---A---GGGG----C-- .

Pf ??????????????????????C---C-GGCC------GAAAGGACC . . . --TG------ ???

Pf $-----A---C-C--T---C C-1--G G C C----A---A A A G G A C C \ldots . . .-C C G---A C G$ ? ? ?

Rp C-a-----C--T--AT-C-CTCCT--G---A--T-C--A- . . . . TTTT--A--T-----Ga--

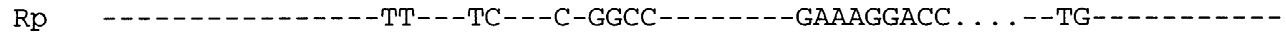

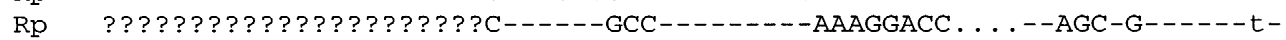

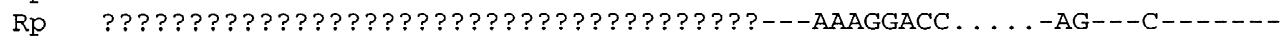

Fig. 1. Variable nucleotide sites from a comparison of all copies of Sry thus far identified in Arvicanthis nairobae (An), Lemniscomys barbarus (Lb), L. massaicus (Lm), L. rosalia (Lr), Pelomys fallax (Pf), and Rhabdomys pumilio (Rp). Sequences are aligned with Sry of inbred mouse strain 129 (Gubbay et al. 1992), which has a Y chromosome of Mus musculus (Мти) origin (Tucker et al. 1992). The sequenced region begins $76 \mathrm{bp} 5^{\prime}$ to the open reading frame and ends 12 bp beyond the stop codon of the Mus musculus sequence. Variable sites

supported by six, four, and two unique and unreversed synapomorphies, respectively.

Within the terminal clades are the putative orthologues for each copy. None of these groups of putative orthologues include representatives from all six species. This reduces our confidence in the identification of orthologues and paralogues, because it leaves open the possibility that a sequence that has diverged from other members of its orthologous clade has been mistakenly identified as paralogous. However, there are many plausible explanations for the apparently missing copies of Sry. For example, one or more copies may have been lost in some lineages, or some duplication events may have been isolated to particular branches of the species phylogeny. It is also likely that our methods did not uncover all of the copies of Sry that occur in these species.

The putative orthologues that are identified here are identical among taxa. This is true even for the CAG repeat region, which was not included in the parsimony analysis.

\section{Relationships Among Putative Paralogues}

Relationships among the seven putative paralogues of Sry are not well resolved. The only strongly supported clade is the grouping of copies 3-7, which is supported by three single-base-pair substitutions and a distinctive 8-bp deletion, followed by a 4-bp insertion. This 12-bp insertion/deletion (indel) was treated as a single charac- within the CAG repeat region were excluded from the figure because they could not be unambiguously aligned. Numbers in vertical columns correspond to the nucleotide positions in Gubbay et al. (1992). The 5' and $3^{\prime}$ untranslated regions are represented by lowercase letters. Dashes indicate sequence identity. Question marks indicate missing data. Dots are gaps in the sequence. There is an 8-bp insertion between positions 8693 and 8694 in several of the sequences.

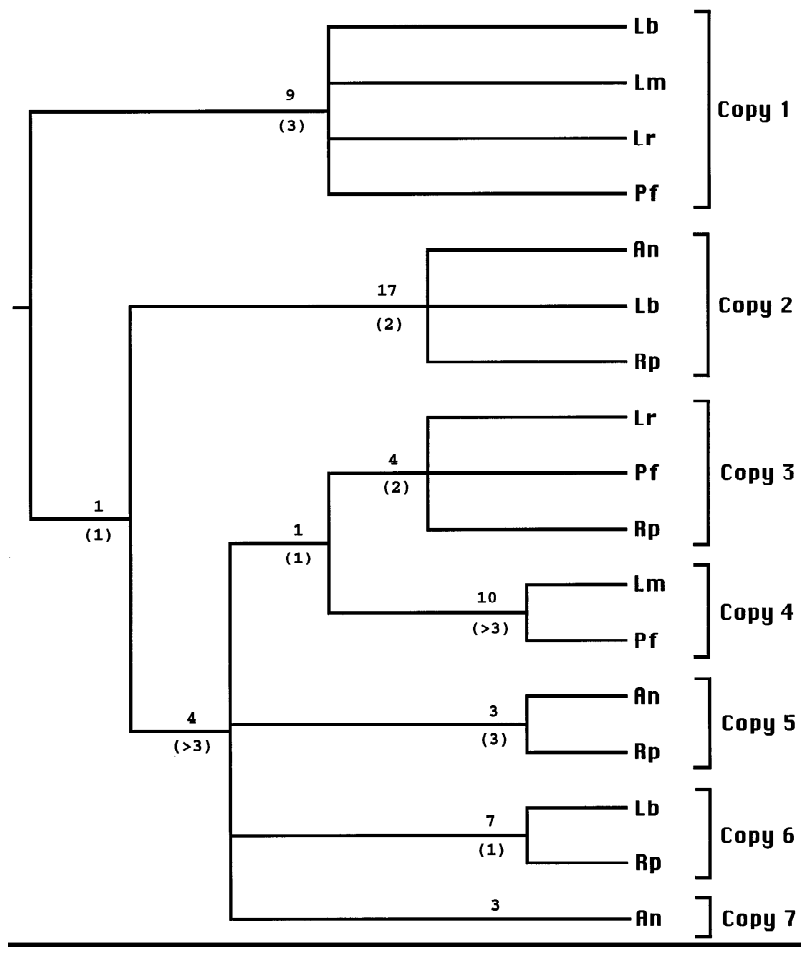

Fig. 2. A strict consensus of 48 trees from a parsimony analysis of the aligned sequences shown in Fig. 1. The Mus musculus Sry sequence was used as an outgroup. Indels were treated as a single character, regardless of their length. Total number of steps $=40, \mathrm{CI}=0.975$. Putative paralogous copies of Sry are labeled as copy 1-7. Number above each branch $=$ unique and unreversed synapomorphies. Number in parentheses below each branch $=$ decay index. Abbreviations for species are the same as in Fig. 1. 
ter in the parsimony analysis. All of the species examined had at least one copy of Sry with this indel, and one copy without it, suggesting that at least one duplication event preceded the speciation event giving rise to this group of rodents.

\section{Pattern of Evolution of Putative Paralogues}

The phylogenetic analysis suggests considerable variation in the rate of nucleotide evolution for different copies of the gene. This cannot be the result of missing data, since there is at least one complete sequence within each terminal clade. An examination of the relative proportion of nonsynonymous to synonymous nucleotide substitutions across the tree revealed a high proportion of nonsynonymous substitutions in both the HMG-box (50\%) and C-terminal regions (70\%). This pattern was not restricted to particular lineages, as might be expected if some copies are free to diverge while others are functionally constrained.

An alignment of the ORFs from the seven putative paralogues of Sry is shown in Fig. 3. The HMG-box is conserved among these copies, with pairwise sequence divergence never greater than $3 \%$. In contrast, the $\mathrm{C}$ terminal region is evolving relatively rapidly. Pairwise sequence divergence reaches 14\% (excluding the repeat) and insertions and deletions result in frameshift mutations that preclude alignment of amino acids beyond the middle of the region. Because of the frameshift mutations, there are two alternative stop codons among the seven copies-one in copies 1,2, and 6 and another in copies 3, 4, 5, and 7. These are located 39 bp and $64 \mathrm{bp}$ $5^{\prime}$ to the putative stop codon of the Mus musculus Sry sequence, respectively.

The repeat region which makes up over half of the ORF of Mus musculus Sry (666 bp) is greatly truncated in all seven of the Sry copies identified here (range 45 to $75 \mathrm{bp}$ ). A shortened repeat region, with respect to the Mus musculus sequence, has been reported in other rodent genera (Tucker and Lundrigan 1993).

\section{Discussion}

The male sex-determining locus, Sry, has duplicated several times in this group of rodents. The duplicate copies are Y-specific, but it is not known whether they are tandemly arranged or dispersed on the chromosome. Duplicate copies of Sry have been detected in other rodent species, including both New World (Bianchi et al. 1993) and Old World (Nagamine 1994) murids. The high incidence of duplicate copies among the few rodent species that have been examined and the occurrence of duplicate copies among rodent species that are not closely related suggest that duplicate copies of Sry are common in rodents.

It is not possible to estimate the age of these gene duplications, as little is known about the phylogenetic history of the rodents examined in this study. However, it is notable that putative orthologues are identical to one another. A comparative study of the evolution of putative orthologues of Sry in the genus Mus (excluding the repeat region) found up to $6 \%$ interspecific sequence divergence (Lundrigan and Tucker 1994). The repeat region has been surveyed in only one species, Mus domesticus, where it exhibited variation among populations (Miller et al. 1995). This suggests that the speciation event that gave rise to the rodent species examined in our study was relatively recent.

In contrast to the lack of variation among orthologues, paralogues have diverged considerably. The most likely explanation for this pattern is that the speciation event that gave rise to the group of rodents examined here was preceded by the gene duplications that gave rise to the seven paralogous copies of Sry isolated from these species. The fact that for each species there is at least one Sry copy with, and one without, the distinctive 12-bp indel is consistent with this hypothesis. However, we cannot eliminate the possibility that one or more of the gene duplications occurred after the origin of the species group and that paralogous copies have diverged more rapidly than orthologous copies. Gene duplication is usually followed by a period of accelerated amino-acid substitution, presumably the result of relaxed constraints on redundant copies or selection for their functional divergence (Li 1985; Ohta 1988, 1994).

Gene duplications have occurred frequently in the evolution of the mammalian genome. The location of Sry on the Y chromosome may make it particularly susceptible to duplication events. Unlike other chromosomes in the genome, the Y chromosome recombines only over a restricted region. Much of the chromosome, including the sex-determining region, is thus clonally inherited from father to son. This unique mode of inheritance has resulted in an unusually rapid rate of duplication, rearrangement, addition, and deletion (reviewed in Marshall Graves 1995). In Mus musculus, Sry lies within $2.8 \mathrm{~kb}$ of unique sequence at the center of a large inverted repeat (Gubbay et al. 1992), a possible relic of multiple copies of Sry in the Mus lineage. Two closely linked genes, zinc finger protein, Zfy (Page et al. 1987), and a gene of unknown function, Ube1Y (Mitchell et al. 1991), have also multiplied on the Mus musculus Y chromosome. Zfy is present in two copies in Mus musculus (Mardon et al. 1989; Nagamine et al. 1989) and has amplified up to 26 times on the $\mathrm{Y}$ chromosome in other rodents (Bianchi et al. 1992; Lau et al. 1992; Nagamine 1994). The two Mus musculus Zfy copies are expressed in the testis and do not appear to be functionally redundant (Koopman et al. 1989).

We do not know whether the Sry copies identified in this study represent functional genes or pseudogenes; the presence of transcripts has not been explored. However, the available evidence suggests that the copies are func- 


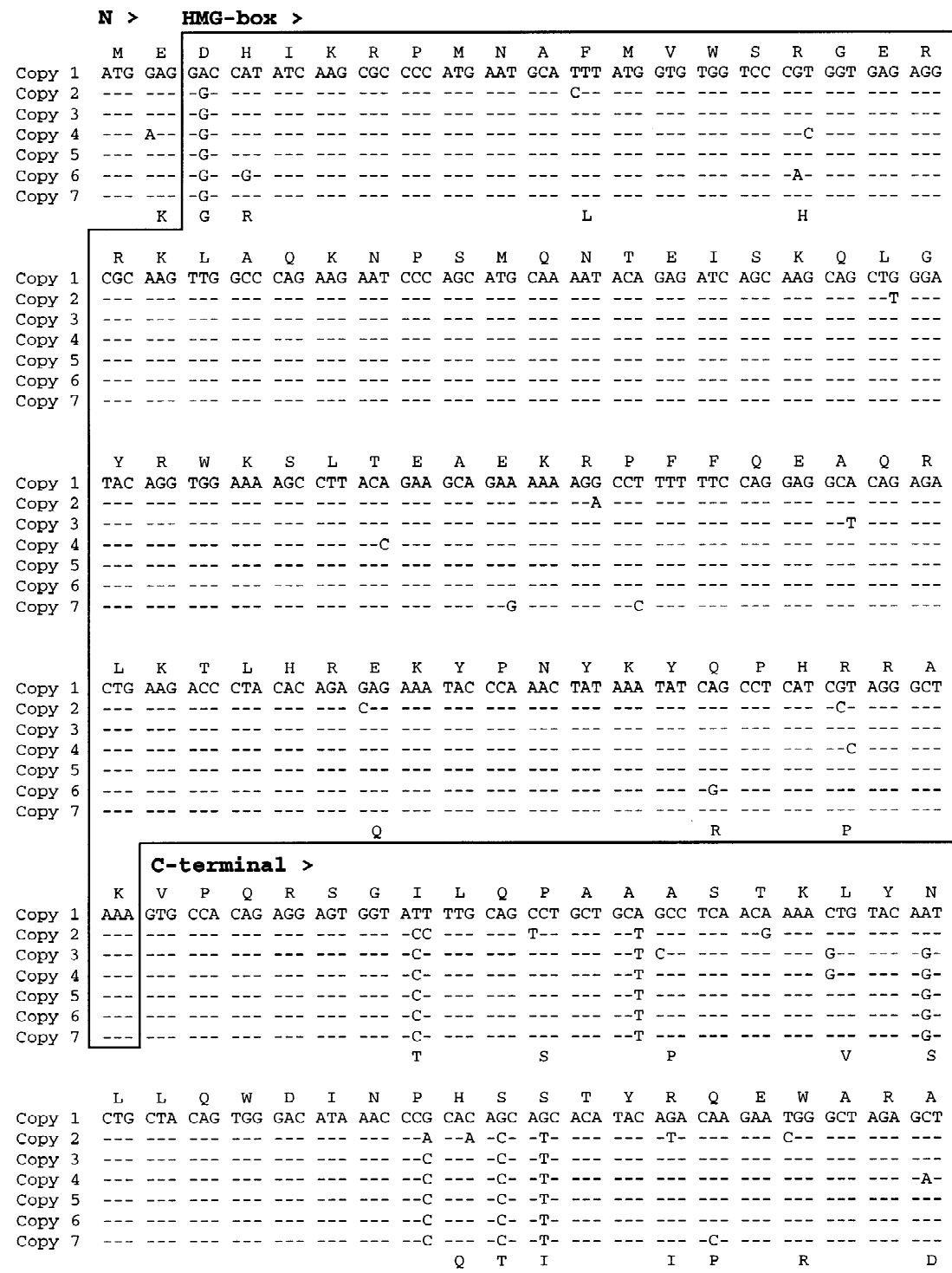

$\begin{array}{lllllllllllllll}A & H & L & S & S & K & A & Q & Q & S\end{array}$

Copy 1 GCA CAC CTG TCC TCC AAG GCC CAG CAA AGC ....... TTTTATTTACAGCCTATGAACATCCCCACTG

COpY 2 _.. _.. -

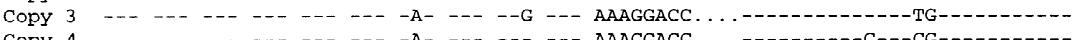

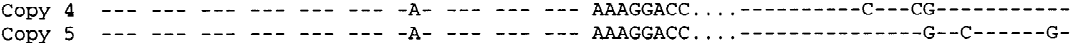

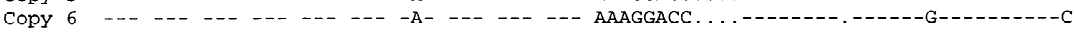

Copy 7 -

repeat

Copy 1 GGCACCCACTGCAGCAGCAG......... CAACAGCAGCAACAGCAGCAGCAATGCCAGCAGCAACACCAGTTCCACCA

Copy 2 -

COpY 3 -

Copy 4 -A-_.

Copy 6

copy 7

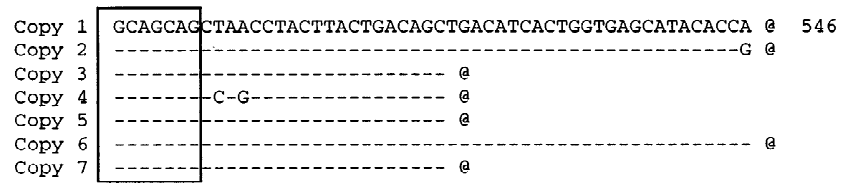

tional. All seven putative paralogues include a long ORF with a conserved HMG-box domain. The pattern of sequence evolution among copies is the same as that previously observed for orthologous comparisons within
Fig. 3. Nucleotide and inferred amino-acid sequences of the ORF from seven copies of Sry that are paralogous according to the phylogenetic analysis shown in Fig. 2. Amino-acid substitutions are indicated below the aligned sequences. The ORF includes an N-terminal region (1-6), HMG-box (7-243, outlined in black), and C-terminal region (244-stop codon, @). Amino-acid sequences could not be aligned beyond nucleotide position 390 because of a 12-bp indel that results in a frameshift mutation. A highly variable CAG repeat motif in the $\mathrm{C}$-terminal region (433-498, outlined in black) could not be unambiguously aligned at the nucleotide level. Dashes indicate sequence identity with copy 1. Dots are gaps in the sequence. Nucleotide 1 corresponds to bp 8304 of the Mus musculus Sry sequence reported in Gubbay et al. (1992). primates (Whitfield et al. 1993) and within rodents (Tucker and Lundrigan 1993): the HMG-box is relatively conserved, while the C-terminal region is evolving rapidly. This indicates that there are different constraints on 
different regions of the Sry copies, a pattern that would not be expected of pseudogenes.

But why should selection conserve redundant copies of Sry? Either there is some advantage to maintaining functionally equivalent, multiple versions of the gene, or these apparently redundant copies have taken on new functions. Inbred mice with two copies of Sry are fertile (Lyon et al. 1981) as are XYY humans (Gorlin 1977). However, there is no obvious selective advantage conferred on individuals that have these duplications. If the copies have acquired new functions, what are they? Clearly, an investigation of gene expression will be needed to answer these questions.

Acknowledgments. We wish to thank W.N. Verheyen for providing the tissue samples used in this study. This research was supported by National Science Foundation grant DEB-9209550 (to P.K.T.).

\section{References}

Bianchi NO, Bianchi MS (1993) Male specific Sry patterns in marsupials. J Mamm 74:531-534

Bianchi NO, Bianchi MS, Pamilio P, Vidal-Rioja L, de la Chapelle A (1992) Evolution of zinc finger-Y and zinc finger-X in OryzomyneAkodontine rodents (Cricetidae). J Mol Evol 34:54-61

Bianchi NO, Bianchi MS, Bailliet G, de la Chapelle A (1993) Characterization and sequencing of the sex determining region $\mathrm{Y}$ gene (Sry) in Akodon (Cricetidae) species with sex reversed females. Chromosoma 102:389-395

Ferrari S, Harley VR, Pontiggia A, Goodfellow P, Lovell-Badge R, Bianchi ME (1992) SRY, like HMG1, recognizes sharp angles in DNA. EMBO J 11:4497-4506

Foster JW, Brennan FE, Hampikian GK, Goodfellow PN, Sinclair AH, Lovell-Badge R, Selwood L, Renfree MB, Cooper DW, Marshall Graves JA (1992) Evolution of sex determination and the Y chromosome: SRY-related sequences in marsupials. Nature 359:531-533

Gorlin RJ (1977) Classical chromosome disorders. In: Yunis JJ (ed) New chromosomal syndromes. Academic Press, New York, pp 60-117

Gubbay J, Collignon J, Koopman P, Capel B, Economou A, Munsterberg A, Vivian N, Goodfellow P, Lovell-Badge R (1990) A gene mapping to the sex-determining region of the mouse $\mathrm{Y}$ chromosome is a member of a novel family of embryonically expressed genes. Nature 346:245-250

Gubbay J, Vivian N, Economou A, Jackson D, Goodfellow P, LovellBadge R (1992) Inverted repeat structure of the Sry locus in mice. Proc Natl Acad Sci USA 89:7953-7957

Hawkins JR (1993) Mutational analysis of SRY in XY females. Hum Mutat 2:347-350

Jenkins NA, Copeland NG, Taylor BA, Lee BK (1982) Organization, distribution and stability of endogenous ecotropic murine leukemia virus DNA sequence in chromosomes of Mus musculus. J Virol 43:26-36

Kallersjo M, Farris JS, Kluge AG, Bult C (1992) Skewness and permutation. Cladistics 8:275-287

Koopman P, Gubbay J, Collignon J, Lovell-Badge R (1989) Zfy gene expression patterns are not compatible with a primary role in mouse sex determination. Nature 342:940-942

Koopman P, Gubbay J, Vivian N, Goodfellow P, Lovell-Badge R (1991a) Male development of chromosomally female mice transgenic for Sry. Nature 351:117-121

Koopman P, Munsterberg A, Capel B, Vivian N, Lovell-Badge R (1991b) Expression of a candidate sex-determining gene during mouse testis differentiation. Nature 348:450-452
Lau Y, Yang-Feng TL, Elder B, Fredga K, Wiberg UH (1992) Unusual distribution of $Z f y$ and $Z f x$ sequences on the sex chromosomes of the wood lemming, a species exhibiting XY sex reversal. Cytogenet Cell Genet 60:48-54

Li W-H (1985) Accelerated evolution following gene duplication and its implication for the neutralist-selectionist controversy. In: Ohta $\mathrm{T}$, Aoki K (eds) Population genetics and molecular evolution. Japan Scientific Societies Press, Tokyo, pp 333-352

Lundrigan BL, Tucker PK (1994) Tracing paternal ancestry in mice, using the Y-linked, sex-determining locus, Sry. Mol Biol Evol 11: 483-492

Lyon MF, Cattanach BM, Charlton HM (1981) Genes affecting sex differentiation in mammals. In: Austin CR, Edwards RG (eds) Mechanisms of sex differentiation in animals and man. Academic Press, New York, pp 329-386

Maddison WP, Maddison DR (1992) MacClade. Analysis of phylogeny and character evolution, version 3. Sinauer, Sunderland, MA

Mardon G, Mosher R, Disteche CM, Nishioka Y, McLaren A, Page DC (1989) Duplication, deletion, and polymorphism in the sexdetermining region of the mouse $\mathrm{Y}$ chromosome. Science 243:78-80

Marshall Graves JA (1995) The origin and function of the mammalian $\mathrm{Y}$ chromosome and Y-borne genes-an evolving understanding. Bioessays 17:311-320

Miller KE, Lundrigan BL, Tucker PK (1995) Length variation of CAG repeats in Sry across populations of Mus domesticus. Mamm Genome 6:206-208

Misonne X (1969) African and Indo-Australian Muridae, evolutionary trends. Tervuren Belgium Musee Royal De L'Afrique Centrale Annales. Sci Zool 172:1-219

Mitchell MJ, Woods DR, Tucker P, Opp JS, Bishop CE (1991) Homology of a candidate spermatogenic gene from the mouse $\mathrm{Y}$ chromosome to the ubiquitin-activating enzyme E1. Nature 354:483-486

Nagamine CM (1994) The testis-determining gene, SRY, exists in multiple copies in Old World rodents. Genet Res Camb 64:151-159

Nagamine CM, Chan K, Kozak CA, Lau Y (1989) Chromosome mapping and expression of the putative testis-determining gene in mouse. Science 243:80-83

Nasrin N, Buggs C, Kong XF, Carnazza J, Goebl M, AlexanderBridges M (1991) DNA-binding properties of the product of the testis-determining gene and related protein. Nature 354:317-320

Ohta T (1988) Further simulation studies on evolution by gene duplication. Evolution 42:375-386

Ohta T (1994) Further examples of evolution by gene duplication revealed through DNA sequence comparisons. Genetics 138:1331-1337

Page DC, Mosher R, Simpson EM, Fisher EMC, Mardon G, Pollack J, McGillivray B, de la Chapelle A, Brown LG (1987) The sexdetermining region of the human $\mathrm{Y}$ chromosome encodes a finger protein. Cell 51:1091-1104

Pallsboll PJ, Vader A, Bakke I, El-Gewely MR (1992) Determination of gender in cetaceans by the polymerase chain reaction. Can J Zool 70:2166-2170

Sanger FS, Nicklen S, Coulson AR (1977) DNA sequencing with chain terminating inhibitors. Proc Natl Acad Sci USA 74:5463-5467

Sinclair AH, Berta P, Palmer MS, Hawkins JR, Griffiths BL, Smith MJ, Foster JW, Frischauf A, Lovell-Badge R, Goodfellow PN (1990) A gene from the human sex-determining region encodes a protein with homology to a conserved DNA-binding motif. Nature 346: 240-244

Swofford DL (1993) Phylogenetic analysis using parsimony (PAUP), version 3.1.1. University of Illinois, Champaign, IL

Tucker PK, Lundrigan BL (1993) Rapid evolution of the sex determining locus in Old World mice and rats. Nature 364:715-717

Tucker PK, Lee BK, Lundrigan BL, Eicher EM (1992) Geographic origin of the Y chromosome in "old" inbred srains of mice. Mamm Genome 3:254-261

Whitfield LS, Lovell-Badge R, Goodfellow PN (1993) Rapid sequence evolution of the mammalian sex-determining gene SRY. Nature 364:713-715 